\title{
TRAINING PROTOCOL FOR POWERED SHOULDER PROSTHESIS
}

\author{
Linda Resnik, PT, PhD; Shana Lieberman Klinger, MA; Kathryn Korp, OTR/L, OTD; Lisa Smurr Walters, ORT/L, CHT
}

\section{INTRODUCTION}

( here is a scarcity of literature on rehabilitation training protocols for upper-limb prosthesis use. Seminal textbooks and articles describe protocols for training individuals to use conventional body-powered, myoelectric, or passive prosthetic devices and were written prior to the introduction of multifunction prosthetic hands and more advanced prosthetic technologies [1-3]. Furthermore, detailed descriptions of training protocols for individuals with shoulder disarticulations or forequarter amputations are particularly limited, with little emphasis on this level of amputation [4]. Two case studies described rehabilitation at this amputation level using cosmetic or body-powered prostheses with single degree of freedom (DOF) terminal devices [5-6]. In 2008, Smurr et al. published an updated training protocol for people with upper-limb amputation that highlighted the process of controls training for body-powered and myoelectric prosthesis users by level of amputation [7]. Stubblefield et al. presented an occupational therapy training protocol specific to patients undergoing targeted muscle reinnervation surgery [8].

Previously published prosthetic rehabilitation protocols do not address prosthetic training with the advanced prostheses that have become available and include multifunction hands, powered prostheses with multiple DOFs, and/or powered shoulder joints. The most widely tested advanced, multifunction prosthesis to date is the DEKA Arm, developed through the Defense Advanced Research Projects Agency's (DARPA's) Revolutionizing Prosthetics program. The DEKA Arm is now in its third generation prototype (Gen 3 ) and was recently approved by the Food and Drug Administration for commercial marketing. The DEKA Arm is modular and can be configured for three levels: radial configuration $(\mathrm{RC})$ for people with transradial amputation, humeral configuration $(\mathrm{HC})$ for people with transhumeral amputation, and shoulder configuration (SC) for people with transhumeral amputation with very short residuum and persons with amputations at the shoulder disarticulation and interscapulothoracic (forequarter) level. Features of the SC DEKA Arm with end-point control and foot controls were described in detail in three articles [9-11]. The SC Arm is shown in Figure 1. Briefly, the SC Arm has 10 powered DOFs and additional passive DOFs. Prosthetic movements are customized for the user and can employ a combination of foot controls, myoelectric controls, pneumatic bladders (provided by DEKA), or other commonly available prosthetic input elements. All levels of the DEKA Arm use control inputs for the hand and wrist. The control scheme has dual modes enabling the user to switch between a "hand mode" of operation for fine motor use and an "arm mode" of operation to control larger gross movements of the prosthetic arm. Gross movements are operated through endpoint control, which allows simultaneous, coordinated movement of multiple joints to move the terminal device (the end point) to a desired position in space.

The Department of Veterans Affairs (VA) has led a study to optimize the DEKA Arm (Optimization Study) since 2008 and is currently leading a study of home use of the device. A synopsis of the general training protocol used in the Optimization Study was presented in two articles [12-13]. Another article describing the virtual reality environment (VRE) portion of the training protocol for the DEKA Arm second generation prototype (Gen 2) reported that VRE training was particularly valuable for people with upper-limb amputation who must master 


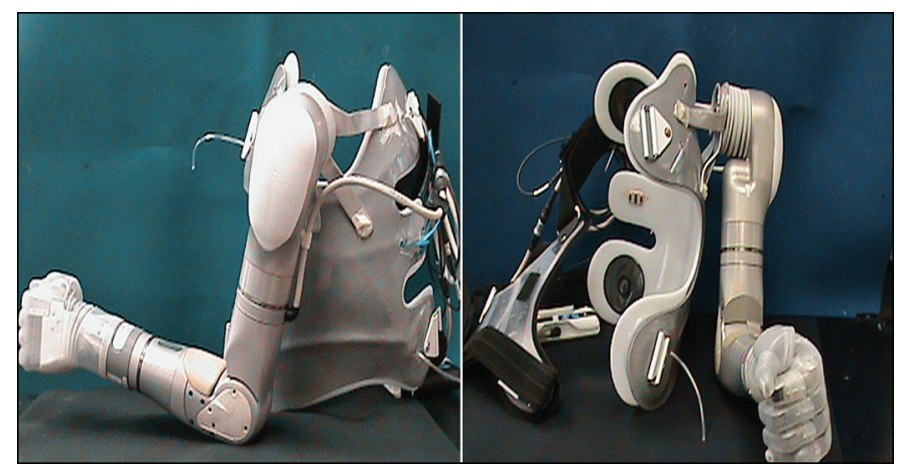

Figure 1.

Shoulder configuration of DEKA Arm.

a large number of controls and for those who need a structured learning environment [14]. This article also reported on the experiences of a single SC Gen 2 user during VRE training [14]. A brief case-example was included in an additional article that described endpoint control for a single SC subject using the Gen 2 DEKA Arm with end-point control and earlier prototypes of inertial measurement unit (IMU) foot controls. The user's control setup and training time were discussed [11]. A separate article reported Optimization Study participants' reactions to and experiences with learning foot controls [10].

Despite these multiple publications to date, the detailed and unique aspects of the Gen 3 SC DEKA Arm training protocol have not been reported. Yet when the DEKA Arm becomes commercially available, therapists will need guidance in developing and implementing effective training strategies for patients learning to use the device. Hence, the purpose of this editorial is to explain the evolution of the training protocol and describe each component of the training guidelines for the Gen 3 SC DEKA Arm.

\section{EVOLUTION OF SHOULDER CONFIGURATION DEKA ARM TRAINING PROGRAM}

During the Optimization Study, the coordinating site and the participating clinicians sites reflected on their experiences working with this technologically complex, multi-DOF prosthetic device, and our team shared results and experiences with the scientific and clinical community. Key lessons from the clinician researcher experiences, such as the need for close prosthetist/therapist collaboration and the need to be sensitive to cognitive burden, were summarized in a previous publication [15]. As of October 2014, the VA research team had trained 48 unique persons to use the DEKA Arm, 12 of whom were trained to use the SC level. These numbers include participants in the Optimization Study and ongoing Home Study.

The DEKA Arm training protocol was developed at the outset of the Optimization Study in $\mathbf{2 0 0 8}$ and was used to guide training sessions. The initial protocol was developed using previously published guidelines on people with upper-limb amputation rehabilitation [1], as well as consultation with DEKA engineers who previously trained several persons with amputation in use of the DEKA Arm and an occupational therapy expert in the field. At the outset of the study, the Gen 2 SC DEKA Arm was operated by direct control, meaning that each powered DOF was operated separately. Thus, the first user was trained to utilize direct control. Within the first $6 \mathrm{mo}$, DEKA introduced the first prototype of end-point control, and the training protocol for Gen 2 SC users was modified accordingly. The training protocol was also modified over the course of the $3 \mathrm{yr}$ Optimization Study as key features of the DEKA Arm evolved, such as foot controls [10], end-point control [9], and hardware [9].

Throughout the Optimization Study, all training sessions were videotaped and the data analyzed. Observations of training challenges (such as teaching end-point trajectories and safety measures) and training techniques that seemed to work well were summarized by the principal investigator and analytical staff. These summaries were provided as informal feedback to occupational therapists (OTs) on an ongoing basis, and broadly applicable training strategies were integrated into successive iterations of the training protocol. Based on these findings, the protocol was refined and the training methods standardized.

At the conclusion of the Optimization Study, the content of the training protocol was reviewed again and refinements and changes were integrated into the current version of the training protocol. This training protocol is used in the VA's current study of the DEKA Arm, the Home Study of an Advanced Upper Limb Prosthesis (Home Study), which is currently ongoing. We do not foresee additional revisions to the protocol during the current study.

\section{Training Protocol Overview}

The Home Study training protocol is divided into three major components: prosthetic controls setup and 
orientation, preprosthetic training, and training with the DEKA Arm. Controls setup and orientation involves the prosthetist and patient establishing a control scheme for prosthetic operation as well as education on how to control the device. Preprosthetic training includes instruction about features of the device and simulated use in a VRE. Training involves use of the DEKA Arm beginning with simple movement activation drills and grasp and release activities to performing increasingly complex unilateral and bilateral daily and recreational activities.

\section{Prosthetic Control Setup and Orientation}

The first step in the training process is prosthetic fitting and basic controls setup. During this phase, the prosthetist and user determine the initial prosthetic control scheme and identify control methods that can include a combination of myoelectric control, foot controls, pneumatic bladders, switches, and linear transducers. The prosthetist configures the DEKA Arm using a specific computer application called the Prosthetist Interface (PI) software. This program allows the prosthetist to associate a selected control method with a user's specific physical action and link both to a featured prosthetic movement of the DEKA Arm. Additionally, the software affords the prosthetist and/or therapist the ability to adjust gains and thresholds or activation sensitivity for each control method. Whenever possible, the OT is present for this process because a thorough understanding of the controls setup is integral to properly train the subject. The subject practices activating each control and identifying the associated action until he/she clearly understands the control for each Arm action.

Detailed information on the features of the DEKA Arm controls is presented elsewhere [9]. Briefly, the HC and SC DEKA Arms have dual control modes so that the user can switch between operation of "hand mode" (to control movements of the hand and wrist) and "arm mode" (to control movements of the powered elbow, shoulder, or combined movements, i.e., end-point control). Up to three movements of the hand and or wrist may be programmed within arm mode if sufficient control inputs are available. The RC DEKA Arm has a single mode. Users of all levels select the grip (from the 6 available) by toggling through the grips in a specified order using the control input signal(s) assigned for grip selection. Some users are set up to toggle forward as well as backward in grip order.

A visual picture of the controls is created and given to the user, who is instructed to review the controls handout each night until he/she demonstrates consistent memory recall for device control and the staff feels confident that the subject has mastered this knowledge. As needed, the initial control setup and/or configuration may be modified by either the prosthetist or the OT during the training process based on user preference and improved skill with control of the DEKA Arm.

\section{Preprosthetic Training}

The next segment within the training protocol is preprosthetic training, which includes instruction about the features of the device and simulated use of the DEKA Arm within a VRE. VRE and simulated use are done with a deactivated DEKA Arm and an avatar on the VRE screen. The protocol calls for a minimum of $4 \mathrm{~h}$ of such training for SC DEKA Arm users. These $4 \mathrm{~h}$ are used to introduce basic information and vocabulary associated with the DEKA Arm such as proper names of each prosthetic component and available device features, movements, and grips. A synopsis of the content of these sessions is shown in Figure 2. The user is taught to recognize the names of the six hand grips and finger postures, potential activities each grip may be used to perform, which grips have built-in "detents" or stopping points in midgrip, and program order of each of the hand grips. The detent feature lets users separate the positioning/stabilizing and grasping aspects of grip from the precision portion and is meant to minimize unintentional finger movements while grasping, releasing, or manipulating a given object. Also, the user is taught the location and function of all user-notification mechanisms such as the LED (light-emitting diode) wrist display and what each light indicates. The subject is also trained on the purposes of the tactor vibrations for grip and mode select and to operate the standby and mode control switches.

Preprosthetic training also includes instruction in the basic principles of IMU use. The IMUs are clipped onto the user's shoes and are used to control the DEKA Arm (Figure 3). The therapist reviews with the user the foot/IMU movements and the associated actions of the DEKA Arm for prosthesis control. The foot movements are reviewed for both hand and arm modes, and the therapist quizzes the user about the control setup while the Arm and VRE are powered off. Important 


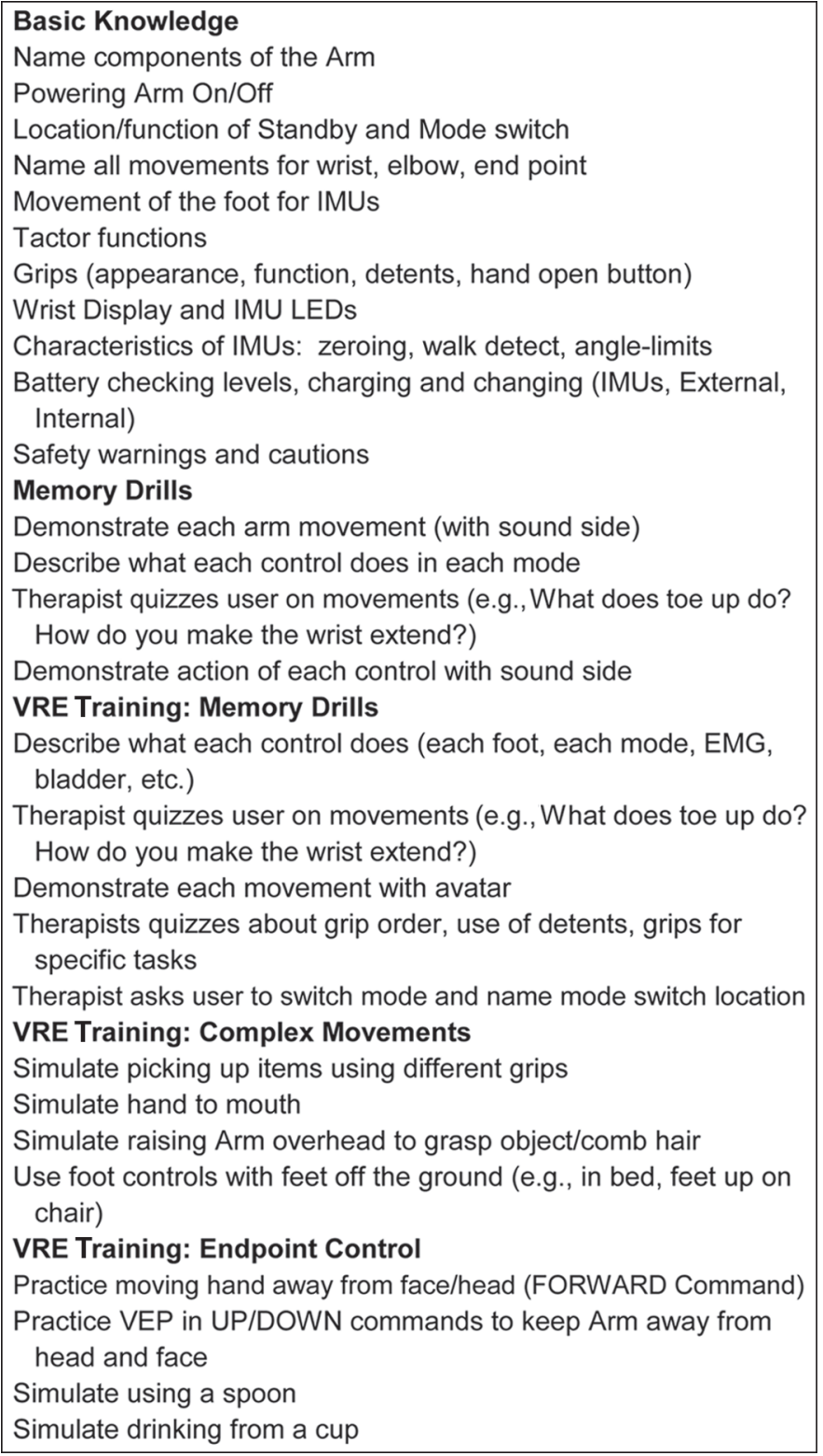

\section{Figure 2.}

Content of preprosthetic training. $\mathrm{EMG}=$ myoelectric control, IMU = inertial measurement unit, LED = light-emitting diode, $\mathrm{ROM}=$ range of motion, VEP = voluntary elbow positioning, VRE = virtual reality environment.

principles covered during IMU training and use include keeping the feet still while at rest to minimize unintended movements of the prosthesis, avoiding extreme foot movements that lead to an "over-angle" detection (in which the foot moves to an extreme angle and foot movements no longer command the specific prosthetic action), correct positioning of the feet when rezeroing the IMUs (rezeroing is done to reset the IMU position

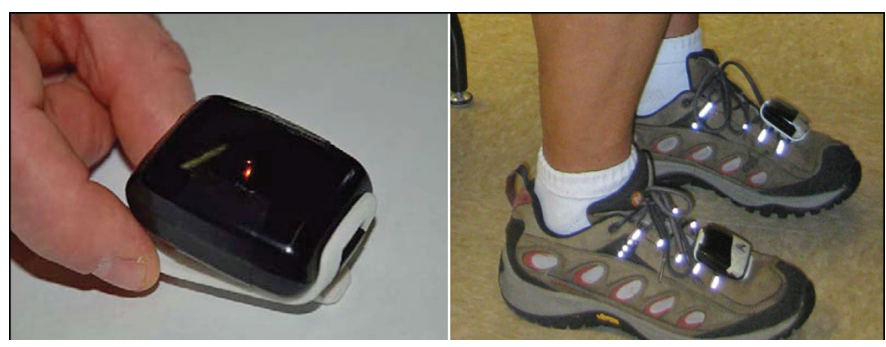

Figure 3.

Inertial measurement unit.

to the starting position), meaning of the built-in LED lights on the IMUs, and the features and function of walk detect.

After basic information is taught, the VRE is powered on and memory drills related to basic Arm functions are repeated. The user controls operation of the various prosthesis functions that are displayed with an avatar on a computer screen [14]. A synopsis of the memory drills used in preprosthetic training is shown in Figure 4. Use of VRE also enables users to become familiar with the abilities and motion trajectories of the DEKA Arm without being distracted by the sound of the motors or movements of the prosthesis in space [14]. SC users practice control of all movements of the DEKA Arm with the VRE system. Specific attention is given to movements for end-point control operation, with focus on movement trajectory of each command (up/down, left/right, forward/back) and the available range of motion produced with the prosthesis. Users are taught basic safety strategies when utilizing the device near the face, such as moving the prosthesis away (arm forward) and experimenting with the slow movement feature, which is a built-in safety mechanism of the DEKA Arm. Other general safety rules for using the DEKA Arm are introduced. Users are asked to experiment with choosing and using different grip patterns, observing the function of each grip. The user is asked to practice using proportional control by performing the hand open and close movements at different speeds or stopping midway.

If myoelectric controls are used at the SC level (which was done in our studies only for persons who were already experienced myoelectric users), electrodes are typically aligned on the large pectoralis major or upper trapezius muscles. If myoelectric controls are chosen for patients who are not experienced users, they will need time and practice to acclimate 


\section{Basic Knowledge Training}

- Therapist names an Arm action and the user demonstrates the action on the sound side (if a person with unilateral amputation) to ensure understanding of the movement name.

- Therapist asks the user to describe the controls governed by the IMUs on each foot and describe the controls of any other inputs in each mode.

$O$ in hand mode

$O$ in arm mode

- e.g., "Tell me what movements are controlled by the right foot in arm mode."

- Therapist quizzes the user on specific movements of the foot sensors and the associated prosthesis movement.

○ e.g., "Tell me what movement happens when you point your right toes down."

- IMU training: Therapist asks the user to demonstrate with the sound hand (if a person with unilateral amputation) what each foot movement (or other control site) does. Therapist makes corrections and provides demonstrations as necessary. The user should verbally state the input and desired motion so there is reinforcement of the controls in BOTH words and actions.

- e.g., "Show me the movements that are controlled by the right foot by using your sound arm (if a person with unilateral amputation)."

- In-depth teaching about grips

- Ask the user to mimic the grip on the sound side to be sure that he/she understands what it looks like.

- Therapist suggests the appropriate grip to be used to perform specific tasks.

- Therapist demonstrates each of the grips using a variety of objects (ball, paper clip, drill, etc.).

- Therapist asks the user to use sound hand to select a grip for each item.

- Asks the user to name the grip.

- Therapist asks user to state which grips have the detents, and explain their use.

- Therapist quizzes user on order of grips (if toggle select) or location (if direct select).

\section{VRE Training}

- Ask the user to demonstrate use of each movement of the DEKA Arm and explain what s/he did to create the movement.

o e.g., "Tell me what you do to make the wrist flex." If the user is unclear about a particular movement, ask him/her to demonstrate the action on the sound side to be sure that the terminology of movement is understood.

- Ask the user to switch mode and name the location of the mode select switch.

- Ask the user to select and use each grip in a sequential manner.

Figure 4.

Specific memory drill activities used in preprosthetic training. IMU = inertial measurement unit, VRE = virtual reality environment.

to these controls, develop skill to isolate the muscles, cocontract them (if necessary for functionality), and use them proportionally to control speed of movement.

Users are guided to observe how voluntary elbow positioning (VEP), a separate powered movement not included within end-point control movements but con- trolled by a separate set of controls, affects the trajectory of the end-point movements. VEP elevates or lowers the upper portion of the prosthesis in a movement comparable to shoulder abduction and adduction. It is also important for the user to observe the functional window in which end-point movements 
operate. When outside the functional window, there are built-in stops that cause the arm to stop and not respond to commands or operate as the user intended. Users are taught to recognize and avoid the positions at the extremes of the functional window where the arm may freeze. Users are also shown how to use the manual release hand button, located on the dorsum of the prosthetic hand, in the event that they encounter a situation where the Arm may have frozen and needs to be released. There are some extreme positions where the users may not be able to reach the single, manual release button (currently on the Gen 3 design), and in these cases they must be taught to ask for assistance or doff the prosthesis. Users are guided to perform simulated complex movements with the avatar such as bringing hand to mouth, using a spoon, raising the arm overhead, and simulating combing hair. They are also encouraged to experiment with activating the foot controls in standing and sitting positions, as well as with the feet elevated off the ground to acclimate to the sensation of using the controls in different positions with and without weight-bearing.

The therapist and/or prosthetist should be available throughout training to make adjustments on the PI software to enable optimal tuning of the thresholds and gains and control settings as the user becomes more comfortable with the device. For example, output gains may initially be set low to prevent fast and abrupt movement, but after the user has gained better control of IMUs or myoelectric controls they might be increased, if desired, to increase the speed of movement.

\section{Training with Activated DEKA Arm}

When VRE training is complete and the user is comfortable with basic operations, training with the activated DEKA Arm begins. The amount of time needed for SC users is variable, depending on their prior prosthesis experience and learning abilities. Based on our experience in the optimization study, we found that $30 \mathrm{~h}$ of training was adequate for most first-time prosthesis users; however, some users may require more time. Our Home Study protocol caps training visits at a maximum of $50 \mathrm{~h}$. Training visits can range from 1 to $2 \mathrm{~h}$ in length. To avoid cognitive fatigue, we recommend that 5 min breaks be given as needed, with a minimum of one break for every 30 min of training.

The prosthetist and therapist may need to assist the user in donning and doffing the Arm system during the early training visits. However, the goal of training should be independent donning and doffing of the device. The therapist works with the user and prosthetist to identify any strategies to assist in this process, including assistive devices or special equipment (such as a custom dressing tree).

Training with the activated DEKA Arm begins with a review of the same information introduced during VRE training, reinforcing new knowledge about the Arm's function and controls (Figure 5). Each training session begins with a brief review of the prosthesis control mechanisms and safety features. Frequent repetition is crucially important because of the complexity of the SC device and the number of controls and functions to remember and integrate. Training progresses from simple movement activation drills and repetitive grasp/ release drills to performance of increasingly complex unilateral and bilateral activities such as opening a door with a knob, cutting meat with knife and fork, folding a bath towel, and reaching overhead to grasp an object (Figure 6).

Users are not asked to perform any activities with the DEKA Arm near the face or overhead until the therapist is confident that they have good control over the device. Throughout the training process, safety awareness is emphasized. Because SC users have no proximal limb control, they must be shown how to use the prosthetic controls to move the terminal device away from their face or body if it gets too close inadvertently. In most cases, the DEKA Arm can be moved away from the face or body by using the arm forward command (which is typically associated with a specific foot movement). The arm forward command brings the terminal device forward in space. Unintentional movements of the DEKA Arm can occur if the user activates a control by mistake or too aggressively, the IMUs need to be rezeroed to reset their starting position, or for other reasons. Even with this type of preparation, users can be fearful when the hand approaches their face. Therefore, when attempting activities near the face and head, users wear safety glasses during the early stages of training this feature. The therapist remains within reach to activate the power-off button during movements near the face or mouth, if needed.

As training progresses, less time is spent on controls training and grasp and release activities and more focus is directed to activities of daily living as well as advanced unilateral and bimanual activities. During training activities, the therapist should pay careful attention to the user's posture and the positioning of the prosthesis. Therapists coach users to 


\begin{tabular}{|l|}
\hline Review Basic Knowledge \\
Donning and doffing \\
Name components of Arm system \\
Power on/off; standby; mode switch (if applicable) \\
Tactor functions \\
Name all prosthesis movements \\
Control movements sitting \& standing \\
Wrist display \\
IMU LEDs \\
Characteristics of IMUs, including walk detect \\
Safety: cautions; warnings; safe use \\
Demonstrate manual release button \\
Battery levels/charging/changing \\
Dynamic socket controller (if applicable) \\
Wrist/Hand \\
Pronation/supination of wrist \\
Compound wrist extension/flexion \\
Open/close each grip (practice detent) \\
Recognize grips of the hand \\
Quiz on grip order \\
Quiz on best grip for specific activities \\
Powered Elbow \\
Flexion/extension of elbow \\
Humeral rotation-internal/external \\
Powered shoulder-end- point control \\
Up/down \\
Forward/backward \\
Left/right \\
Elbow in/elbow out (VEP) \\
Safe operation near head \\
Hand to mouth sequence \\
Drills \\
Verbalize each control while performing movement \\
Quiz user on controls \\
Grasp and Release Training \\
Objects midline on table (using different grips) \\
Grasp and release using form board \\
Stacking plastic cups/cones/blocks \\
Shadow boxing \\
\hline
\end{tabular}

Figure 5.

Content of training program with activated DEKA Arm-knowledge review. IMU = inertial measurement unit, LED = light-emitting diode, VEP = voluntary elbow positioning.

avoid compensatory movements when possible and to use the full capabilities of the prosthesis instead. For SC users, training activities include frequent repetition of complex activities, such as the hand-to-mouth sequence for eating and drinking, and frequent rein-

\author{
Activities \\ Drink from a paper cup \\ Grasp spoon, pick up edible object, and bring to mouth \\ Grasp fork, use it to spear object, bring it to mouth, eat \\ Cut meat with knife and fork \\ Brush or comb hair \\ Pour from a 12 oz can or bottle \\ Write a word legibly \\ Open door with knob \\ Use a key in a lock \\ Dial a touch tone phone \\ Use a paper clip \\ Reach overhead to grasp object \\ Put on socks \\ Tie shoe laces \\ Stir cereal in a bowl with a large spoon \\ Use scissors to cut paper \\ Open an envelope \\ Put toothpaste on a brush and brush teeth \\ Put on and remove a T-shirt \\ Button a shirt with front buttons \\ Attach the end of a zipper and zip a vest \\ Carry a laundry basket \\ Use a hammer and nail \\ Put on and take off prosthesis \\ Fold a bath towel \\ Community Outings \\ Eat a meal in public \\ Ride in a car or some type of public transportation, \\ deactivating IMUs \\ Enter a store, select, carry purchases, and pay for items \\ Home Use Preparedness \\ Review of DEKA Arm safety information \\ Review "troubleshooting arm" section in user guide \\ Change the external battery independently \\ Charge batteries (external/internal, IMU) independently \\ Store the Arm independently \\ Pack the Arm for shipment independently \\ Use hand, elbow, and/or shoulder release independently \\ Use Arm without external battery ( $\mathrm{HC}$ and $\mathrm{SC}$ ) \\ Demonstration of Independence
}

\section{Figure 6.}

Content of training program with activated DEKA Arm-activities. $\mathrm{HC}=$ humeral configuration, IMU = inertial measurement unit, $\mathrm{SC}=$ shoulder configuration

forcement of the command for safety, i.e., moving the hand away from the head/face.

Teaching SC users to be able to predict and adjust the trajectories of end-point movements is crucial. Endpoint controls such as up, down, left, right, move the terminal device in space, and the movement pathway of the hand depend on the initial starting position of the elbow and shoulder joints. In addition, users are 
taught how VEP influences the trajectory of the endpoint movements. With practice, the user can learn to anticipate the pathway that the terminal device will take depending upon the starting position. Repeated experimentation using end-point commands with the shoulder and elbow joints positioned at various points in space during movement initiation helps the user understand this operation.

Throughout training, strategies are taught to ensure safety and resolve problems such as freezing or faulting of the Arm. Users are guided to move the device to the extremes of the functional window and experience the result, which is typically faulting or freezing of the Arm. This provides an opportunity for the user to build confidence with troubleshooting. Faulting or freezing at the extremes of motion is resolved by manually releasing and adjusting the joints of the prosthesis, if necessary. Some users find this challenging to do alone, especially if the hand is outstretched fully away from the body. When this is the case, the individual is encouraged to think through and problem-solve situations where the Arm might be frozen in a position that prevents ability to reach the hand open button.

Advanced training includes performance of activities, projects, recreational tasks, or games such as preparing a simple meal of the user's choice, putting a golf ball, or completing a model-building project. Patient-specific activities of special importance to the user are practiced whenever possible. For example, if a user has a desire to use the Arm for sewing, therapy sessions need to include sewing-related activities to build prosthesis skill proficiency.

Training sessions also include time to use the DEKA Arm without any instruction, but still under close supervision by the therapist. This helps the user experiment with arm motions and simple activities of his/her own choosing within reason. During this time, the user is encouraged to try the activity independently without coaching or teaching by the therapist or prosthetist, except as necessary to maintain safety.

Once users demonstrate confidence and comfort with operating the prosthesis, they progress to supervised prosthesis use in a public setting. Our protocol requires a minimum of three community outings that include the following common activities: eating a meal in public; riding in a car or using public transportation; and going to a store to select, carry, purchase, and pay for items. This practice is necessary to desensitize the user to being in the public eye and using a novel device that may draw unexpected attention and scrutiny.

Prior to discharge to the home-use portion of our study, users are required to demonstrate that they are independent with the device by performing a complex activity without any therapist cueing or feedback. In preparation for home use, the therapist must be sure that the user understands basic safety rules when using the device (such as keeping away from open flames, not using the Arm to operate a vehicle, etc.). Users must also be taught to charge and replace all batteries, store the device and components safely, and pack the device for shipment if repair is necessary.

A full discussion of differences between the training protocol for an SC level DEKA Arm and the training protocol for users of the RC and HC DEKA Arm are beyond the scope of this article but are highlighted in the Appendix (available online only).

\section{DISCUSSION}

The training protocol presented in this article is the first of its kind, a detailed example of prosthetic rehabilitative training for individuals receiving a technologically advanced prosthesis after a shoulder disarticulation or forequarter amputation.

This protocol, though specific to the Gen 3 DEKA Arm, demonstrates an approach to training subjects to operate a technologically complex device that has unique control inputs and movement trajectories.

As other new technologically complex upper-limb prosthetic devices become commercially available for users, clinicians will need to develop the expertise to adequately prepare users to operate each device. Our protocol is a well-tested approach for training with this particular prosthesis. However, it also offers a framework for modeling the development of training protocols for other new, technologically complex prosthetic devices. The DEKA Arm training protocol includes training in skilled unilateral movements with the prosthesis. This approach represents a shift away from prosthetic rehabilitation programs that focus solely on use of the prosthesis as an assist. Our view is that an advanced prosthesis, like the DEKA Arm, should assume the role of a nondominant limb, which is capable of skilled activity, though generally less adept than the dominant limb. We believe that key principles, summarized in Figure 7, incorporated into this protocol are generalizable to other advanced prostheses.

\section{CONCLUSIONS}

This article presents a detailed prosthetic training program for users of the SC of the Gen 3 DEKA Arm. This program originated in training protocols developed 
1. Teach the user to understand the device features, its controls, and user notifications.

2. Perform repetitive practice to master the control scheme.

3. Teach the user awareness of proper body mechanics while using the device.

4. Train the user to be aware of potential safety issues related to use of the device.

5. Progress activities from simple to complex.

6. Teach skilled unilateral activities with the prosthesis so that the prosthetic side can function more equivalently to a nondominant upper limb.

Figure 7.

Key principals of prosthetic rehabilitation with advanced prosthesis.

and used in the VA Study to Optimize the DEKA Arm and the VA's Home Study of an Advanced Upper Limb Prosthesis, which is currently ongoing. This article may provide a framework for training protocols for other new, technologically complex prosthetic devices involving multiple DOFs and powered shoulder joints.

\section{ACKNOWLEDGMENTS}

\section{Author Contributions:}

Study concept and design: L. Resnik.

Assisted in training protocol design: S. Lieberman

Klinger.

Center for the Intrepid principal investigator: L. Smurr

Walters.

Subject training: K. Korp, L. Smurr Walters.

Analysis and interpretation of data: L. Resnik,

S. Lieberman Klinger.

Drafting of manuscript: L. Resnik, S. Lieberman

Klinger, K. Korp, L. Smurr Walters.

Obtained funding: L. Resnik.

Funding/Support: This material was based on work supported by VA Rehabilitation Research \& Development (grants VA RR\&D A6780 and VA RR\&D A6780I). DEKA's support of the VA optimization studies was sponsored by DARPA and the U.S. Army Research Office.

Additional Contributions: The authors wish to thank all participating study OTs for their efforts in training subjects and sharing their experiences and recommendations regarding prosthetic training methods.

Disclaimer: The information in this article does not necessary reflect the position or policy of the U.S. Government; no official endorsement should be inferred.

\section{Linda Resnik, PT, PhD; ${ }^{1-2 *}$ Shana Lieberman Klinger, MA; ${ }^{1}$ Kathryn Korp, OTR $/$ L, OTD; ${ }^{3}$ Lisa Smurr Walters, OTR/L, CHT $^{3}$}

${ }^{1}$ Providence VA Medical Center, Providence, RI; ${ }^{2}$ Department of Health Services Policy and Practice, Brown University, Providence, RI; ${ }^{3}$ The Center for the Intrepid, Fort Sam Houston, TX

*Email: Linda.Resnik@va.gov

\section{REFERENCES}

1. Smith DG, Michael JW, Bowker JH, editors. Atlas of amputations and limb deficiencies: Surgical, prosthetic, and rehabilitation principles. Rosemont (IL): American Academy of Orthopaedic Surgeons; 2004. p. 275-84.

2. Meier R, Atkins DJ. Functional restoration of adults and children with upper extremity amputation. New York (NY): Demos Medical Publishing; 2004.

3. Dillingham T. Rehabilitation of the upper limb amputee. In: Office of the Surgeon General. Textbook of military medicine part IV, surgical combat casualty care. Falls Church (VA): TMM Publications; 1995.

4. Johnson SS, Mansfield E. Prosthetic training: Upper limb. Phys Med Rehabil Clin N Am. 2014;25(1):133-51. [PMID:24287244] http://dx.doi.org/10.1016/j.pmr.2013.09.012

5. Canelón MF. Training for a patient with shoulder disarticulation. Am J Occup Ther. 1993;47(2):174-78. [PMID:8470748] http://dx.doi.org/10.5014/ajot.47.2.174

6. Garza P. Case report: Occupational therapy with a traumatic bilateral shoulder disarticulation amputee. Am J Occup Ther. 1986;40(3):194-98. [PMID:3963130] http://dx.doi.org/10.5014/ajot.40.3.194

7. Smurr LM, Gulick K, Yancosek K, Ganz O. Managing the upper extremity amputee: A protocol for success. J Hand Ther. 2008;21(2):160-75, quiz 176. [PMID:18436138] http://dx.doi.org/10.1197/j.jht.2007.09.006

8. Stubblefield KA, Miller LA, Lipschutz RD, Kuiken TA. Occupational therapy protocol for amputees with targeted muscle reinnervation. J Rehabil Res Dev. 
2009;46(4): 481-88. [PMID:19882483] http://dx.doi.org/10.1682/JRRD.2008.10.0138

9. Resnik L, Klinger SL, Etter K. The DEKA Arm: Its features, functionality, and evolution during the Veterans Affairs Study to optimize the DEKA Arm. Prosthet Orthot Int. 2014;38(6):492-504. [PMID:24150930]

10. Resnik L, Lieberman Klinger S, Etter K, Fantini C. Controlling a multi-degree of freedom upper limb prosthesis using foot controls: User experience. Disabil Rehabil Assist Technol. 2014;9(4):318-29.

[PMID:23902465]

http://dx.doi.org/10.3109/17483107.2013.822024

11. Phillips S, Resnik L, Fantini C, Latlief G. Endpoint control for a powered shoulder prosthesis. J Prosthet Orthot. 2013;25(4):193-200. http://dx.doi.org/10.1097/JP0.0000000000000006

12. Resnik L, Latlief G, Klinger SL, Sasson N, Walters LS. Do users want to receive a DEKA Arm and why? Overall findings from the Veterans Affairs Study to optimize the DEKA Arm. Prosthet Orthot Int. 2014;456-66. [PMID:24286806]

13. Resnik L, Klinger SL, Etter K. User and clinician perspectives on DEKA arm: Results of VA study to optimize DEKA arm. J Rehabil Res Dev. 2014;51(1):27-38.
[PMID:24805891]

http://dx.doi.org/10.1682/JRRD.2013.03.0068

14. Resnik L, Etter K, Klinger SL, Kambe C. Using virtual reality environment to facilitate training with advanced upper-limb prosthesis. J Rehabil Res Dev. 2011;48(6): 707-18. [PMID:21938657] http://dx.doi.org/10.1682/JRRD.2010.07.0127

15. Resnik L, Meucci MR, Lieberman-Klinger S, Fantini C, Kelty DL, Disla R, Sasson N. Advanced upper limb prosthetic devices: Implications for upper limb prosthetic rehabilitation. Arch Phys Med Rehabil. 2012;93(4):710-17. [PMID:22464092]

http://dx.doi.org/10.1016/j.apmr.2011.11.010

This article and any supplementary material should be cited as follows:

Resnik L, Lieberman Klinger S, Korp K, Smurr Walters L. Training protocol for powered shoulder prosthesis. J Rehabil Res Dev. 2014;51(8):vii-xvi. http://dx.doi.org/10.1682/JRRD.2014.07.0162

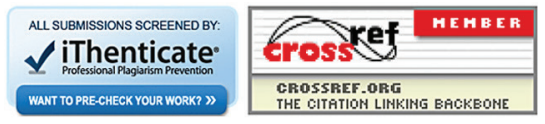

